\title{
Channel-Aware Identification Protocol of Moving Tags in RFID Systems
}

\author{
Ji Hyoung Ahn ${ }^{1}$, Jongho Park ${ }^{2}$, Tae-Jin Lee ${ }^{1 *}$ \\ ${ }^{1}$ College of Information and Communication Engineering, Sungkyunkwan University, Suwon, Korea. \\ 2 Convergence R\&D Lab., LG Electronics, Seoul, Korea. \\ * Corresponding author. Tel.: +82-31-290-7149; email: tjlee@skku.edu \\ Manuscript submitted October 10, 2014; accepted April 25, 2015. \\ doi: 10.17706/ijcce.2015.4.5.354-362
}

\begin{abstract}
In an RFID system, a reader has to be able to identify tags reliably. When the tags have mobility, the reader should identify quickly within a certain limited time to identify all tags. Existing RFID anti-collision protocols do not consider the movement of tags, so there may be some tags that a reader cannot identify. And if tags have mobility, the channel between a reader and tags varies by time. So a reader has to react to the varying channel condition. In this paper, we propose a new anti-collision protocol taking the mobility of tags and channel error into consideration. The proposed anti-collision protocol allows the tags to use the best line code under the moving tag environment. We show that the performance of RFID identification is improved using the proposed protocol via analysis and simulations.
\end{abstract}

Key words: Anti-collision, moving tag, line code, RFID.

\section{Introduction}

Radio Frequency Identification (RFID) is a wireless technology for a reader to access the information stored in tags. In a passive RFID system, a reader transmits continuous wave (CW) to generate the power of tags and the tags transmit their IDs by back-scattering [1], [2]. The back-scattering signals of tags are attenuated significantly by their distances to the reader [3]-[6], and are susceptible to interference or noise [7], [8]. So the received signals of tags are different by the distances to the reader. A reader has to react to the varying channel condition and decide the rate or line code of a tag appropriately. In EPCglobal Class1 Generation2 (C1 G2) [9], 4 types of line codes, i.e., FM0, Miller $M=2,4$ and 8, with different transmission rates are defined. The line code type to be used during an identification round can be specified by a reader in EPCglobal C1 G2. However, how line codes are utilized is not presented in the specification. Moreover, if RFID tags have mobility, the received signals of tags vary by the movement of tags, and some tags may be out of the identification range of a reader before the tags are identified [10]-[12]. So a dynamic line code utilization mechanism is required due to the variation of the signal.

A tag anti-collision protocol for identification of multiple tags requires additional attention to cope with the channel variation and the movement of tags since most of existing tag anti-collision protocols do not consider channel condition and mobility of tags. The anti-collision protocol of EPCglobal C1 G2 is based on Dynamic Framed Slotted ALOHA (DFSA) protocols [13]. The performance of DFSA is influenced by the frame size, but the frame size decision method is not presented in EPCglobal C1 G2. In general DFSA protocols, a reader estimates the number of tags from the slot statistics (numbers of success, idle, and 
collision slots), since the frame size is optimal when the frame size is the same as the number of tags [14], [15]. However most existing tag estimation methods may not be accurate when the tags of a reader have mobility and different channel status. While the identification probability of tags with mobility is considered in [16], [17], a line code decision mechanism for changing channel status is not presented.

In this paper, we propose a new anti-collision protocol taking the mobility of tags and channel error into consideration. The proposed anti-collision protocol can allow the tags to use the best line code under the moving tag environment. The proposed anti-collision protocol quickly identifies tags by high-rate line codes when the tags come closer to the reader. We propose a new frame size decision method according to the speed and the density of tags.

\section{Proposed Identification Protocol}

We consider an RFID monitoring/tracking system, e.g., a conveyor belt RFID system. A fixed RFID reader tries to identify the moving tags on the conveyor belt. The velocity of tags is assumed to be constant. The tags go through the reader's identification range $R$ with the speed of $V_{\text {tag }}$. The density of tags is denoted as $D_{\text {tag }}$, which represents the line density, i.e., the number of tags in a unit length. We assume that the reader knows $V_{\text {tag }}$ and $D_{\text {tag }}$.

There are 4 types of line codes in EPCglobal C1 G2, i.e., FM0, Miller $M=2,4$ and 8. The line code is decided and notified to the tags at the start of an identification round in a Query command. The FM0 has the highest transmission rate, while Miller $M=2$ has the half of the FM0's transmission rate. Miller $M=4$ has slower rate than that of Miller $M=2$, and the rate of Miller $M=8$ is the half of that of Miller $M=4$. If tags are static or the channel condition is good, it is appropriate that a reader selects FM0. In the proposed monitoring/tracking system model, tags move on the line-type belt. That is the tags which come into the reader's identification range approach and pass the reader, and then leave out of the reader's identification range. In an RFID system with moving tags, the distances between the tags and the reader vary within the identification range of a reader. And the channel conditions of tags also change.

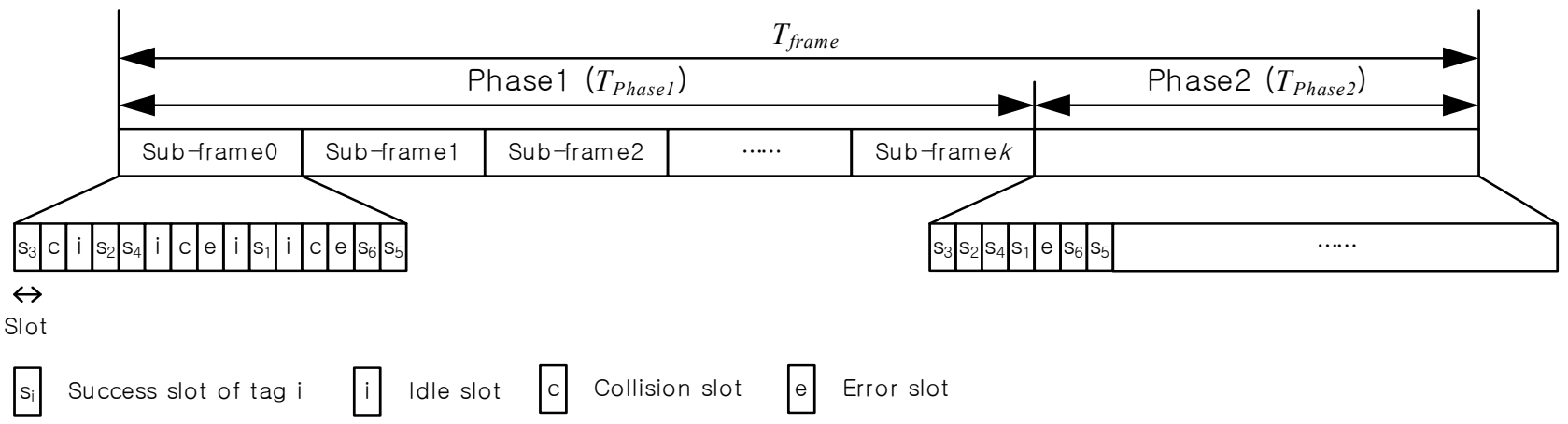

Fig. 1. Frame structure of the proposed protocol.

The frame structure of our proposed anti-collision protocol consists of 2 phases (see Fig. 1). In Phase 1, a reader operates a DFSA protocol in the sub-frames to determine the transmission orders of tags in Phase 2. The tags transmit 16-bit random numbers (RN16) [9] in a sub-frame, not transmitting actual IDs (PC+EPC+CRC16). When the reader receives an RN16, it sends an ACK with the same RN16 as the reader received, and a counter value to designate the order of transmission of actual IDs in Phase 2. The tag receiving the ACK transmits the same counter value as that in the reader's ACK. The reader then progresses the slot after it receives the tag's counter value. If there is a collision by more than one tag when RN16s are transmitted, the RN16s may be corrupted. Then the collided tags do not reply to the reader's ACK and try to transmit their RN16s at the next sub-frame. Fig. 2 shows an example of Phase 1 of the proposed 
anti-collision protocol. In Phase 1, tags transmit RN16s and counter values using the most reliable line code (Miller $M=8$ ) to acquire the transmission opportunity since the tags may not be close enough to the reader.

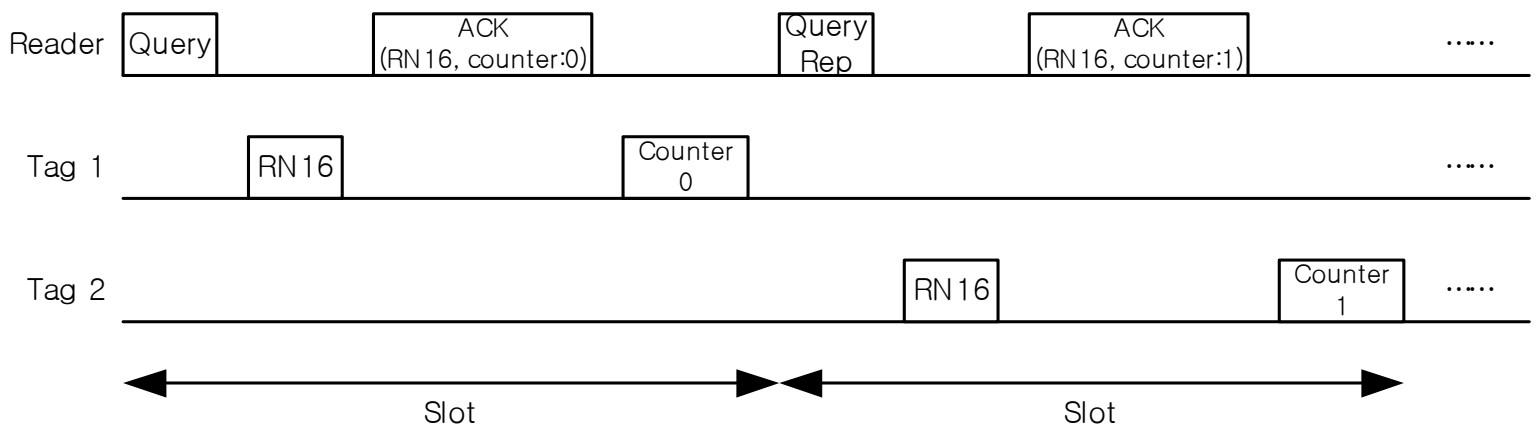

Fig. 2. An example of Phase 1 of the proposed protocol.

When tags close enough to the reader to use high-rate line codes, Phase 2 starts. In Phase 2, a reader identifies the tags which have successfully transmitted RN16s at Phase 1 using the fastest line code (FM0). The tag with counter 0 transmits its ID, and the other tags decrease their counters by 1 when they listen to the ACK. Note that the order of transmission of IDs is decided at Phase 1. So there is no collision in Phase 2, although channel error may occur. If the reader cannot decode the ID of a tag, the reader transmits NACK. Then the tag which receives NACK with counter 0 retransmits its ID. Fig. 3 shows an example of Phase 2 of the proposed anti-collision protocol.

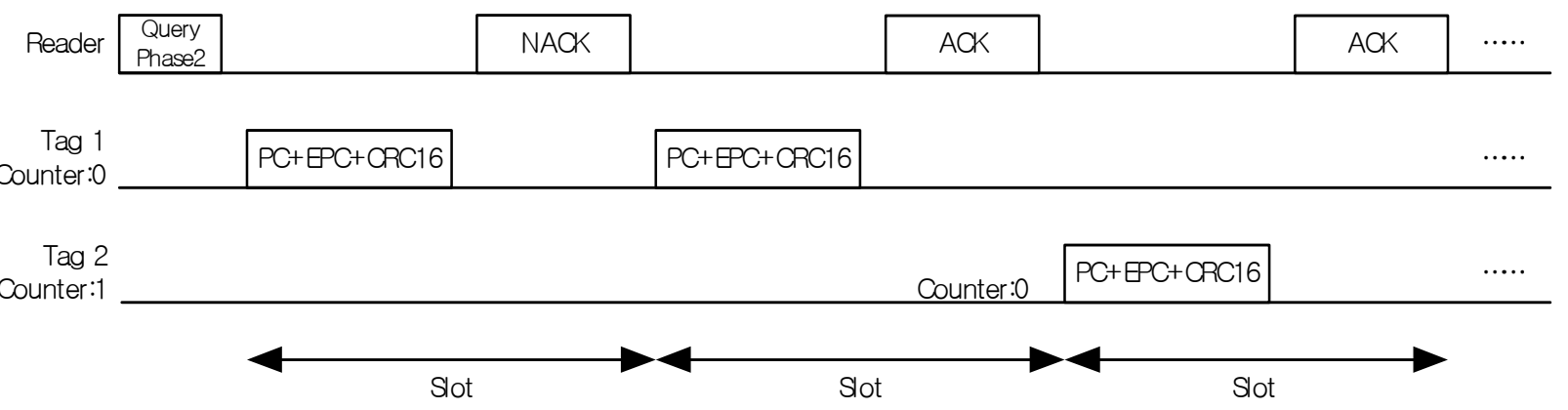

Fig. 3. An example of Phase 2 of the proposed protocol.

Our proposed anti-collision protocol uses FM0 line code in Phase 2. To guarantee the desired Packet Error Rate (PER) in Phase 2, we present constraints on the frame size. The reader identifies tags which come into the reader's identification range. If the required PER is $P E R_{r e q}$, the distance $d_{F M 0}$ which can achieve the required PER $P E R_{r e q}$ using FM0 can be computed by solving the following (1) (3).

$$
\operatorname{PER}\left(d_{F M 0}\right)=1-\left(1-\operatorname{BER}\left(E\left(d_{F M 0}\right)\right)\right)^{m}=P E R_{r e q}
$$

The bit error rate (BER) is given in [18].

$$
\begin{aligned}
& \operatorname{BER}\left(E\left(d_{F M 0}\right)\right) \\
& =2 Q\left(\sqrt{2^{k} \frac{E\left(d_{F M 0}\right)}{N_{0}}}\right)\left(1-Q\left(\sqrt{2^{k} \frac{E\left(d_{F M 0}\right)}{N_{0}}}\right)\right)
\end{aligned}
$$


where $E\left(d_{F M 0}\right)$ is the energy of the received signal when FM0 is used, $N_{0}$ is the noise power spectral density, $Q(\cdot)$ is the $Q$ function, and $k$ is the line code index ( $k=0$ for FM0, $k=1,2,3$ for Miller $M=2,4,8$ ). The received signal power at the distance of $d$ is

$$
P_{R X}(d)=\alpha \frac{P_{T X} \times G_{\text {reader }}^{2} \times \lambda^{4} \times G_{\text {tag }}^{2}}{(4 \pi d)^{4}} \times 0.25
$$

where the factor $\alpha$ denotes the degree of the load impedance mismatch on the amount of re-radiated power. The factor $\alpha$ is 1 since we consider the antenna with a complex conjugate-matched load [3]. And $P_{T X}$ is the transmission power of a reader's continuous wave, $\lambda$ is the wavelength of a reader's continuous wave, $d$ is the distance between a reader and a tag, and $G_{\text {reader }}$ and $G_{t a g}$ are the antenna gains of a reader and a tag, respectively. The factor 0.25 denotes the portion of the reflected tag signal power among the total received power at a tag using ASK modulation.

The tags at Phase 1 have to be identified (by RN16) within $R-d_{F M 0}$ to use FM0 at Phase 2. So the size of Phase $1 T_{\text {Phase } 1}$ has to satisfy the following relationship.

$$
T_{\text {Phase } 1} \geq\left(R-d_{F M 0}\right) / V_{\text {tag }}
$$

And the tags have to be within $R+d_{F M 0}$ during the identification process at Phase 2 . So the maximum frame size $T_{\text {frame }}$ has to satisfy the following equation.

$$
2 T_{\text {frame }} \leq\left(R+d_{F M 0}\right) / V_{\text {tag }} .
$$

The size of Phase $2 T_{\text {Phase } 2}$ can be computed by

$$
T_{\text {Phase } 2}=n \times T_{\text {slot-phase } 2} \times\left(1-P E R_{\text {req }}\right)^{-1}+T_{\text {guard }}
$$

where $T_{\text {slot-phase } 2}$ is the size of a slot in Phase 2 and $T_{\text {guard }}$ is a guard time for the tags experiencing unexpected channel error. The number of tags $n$ which newly come into the reader's identification range during a frame is

$$
n=T_{\text {frame }} \times V_{\text {tag }} \times D_{\text {tag }} .
$$

And the frame size is determined as

$$
T_{\text {frame }}=T_{\text {Phase1 }}+T_{\text {Phase 2 }} .
$$

The frame size should be decided to satisfy (5). Then the size of Phase 2 can be computed by (6). Next the size of Phase 1 is decided by (4) and (8). These constraints of time parameters are shown in Fig. 4 and 5. 

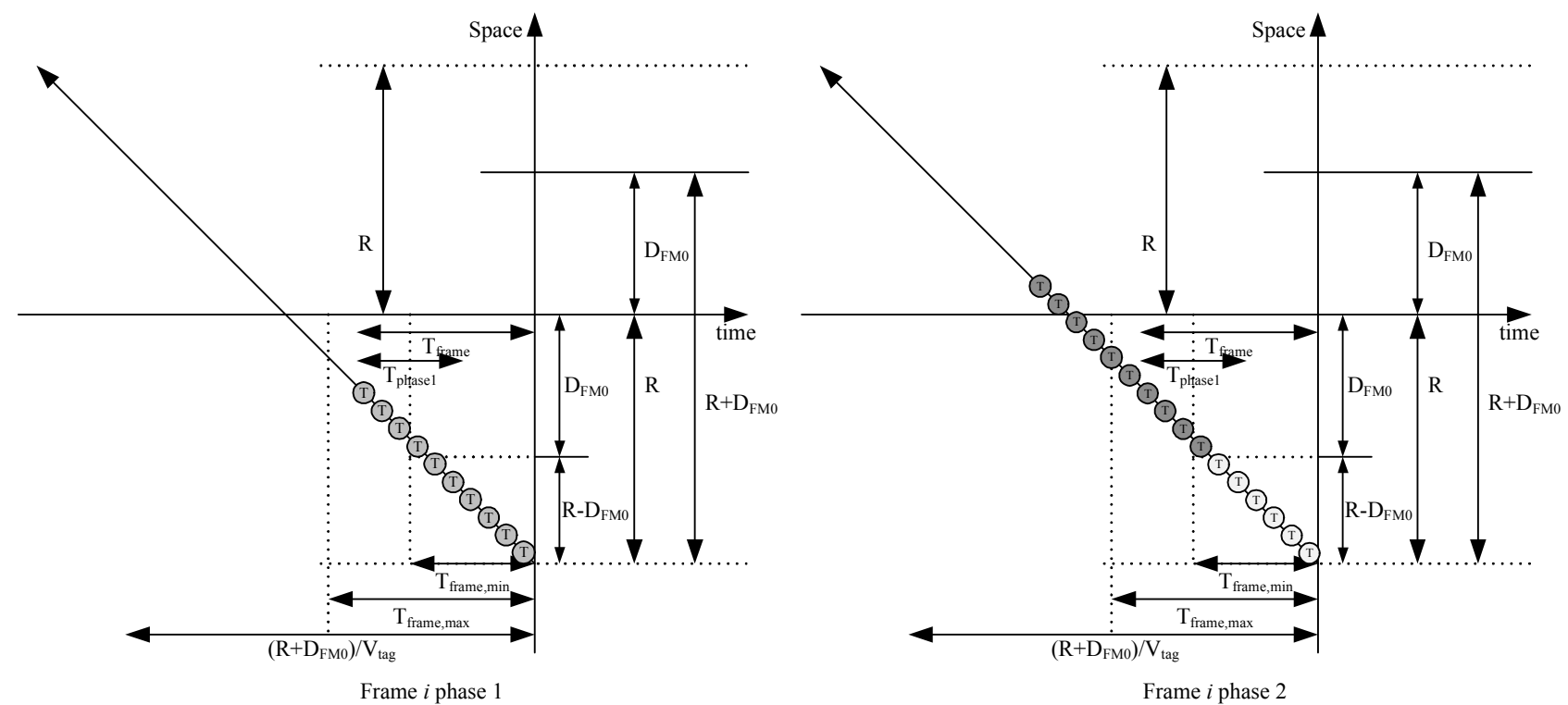

Fig. 4. An example of the proposed protocol at the $i$ th frame.
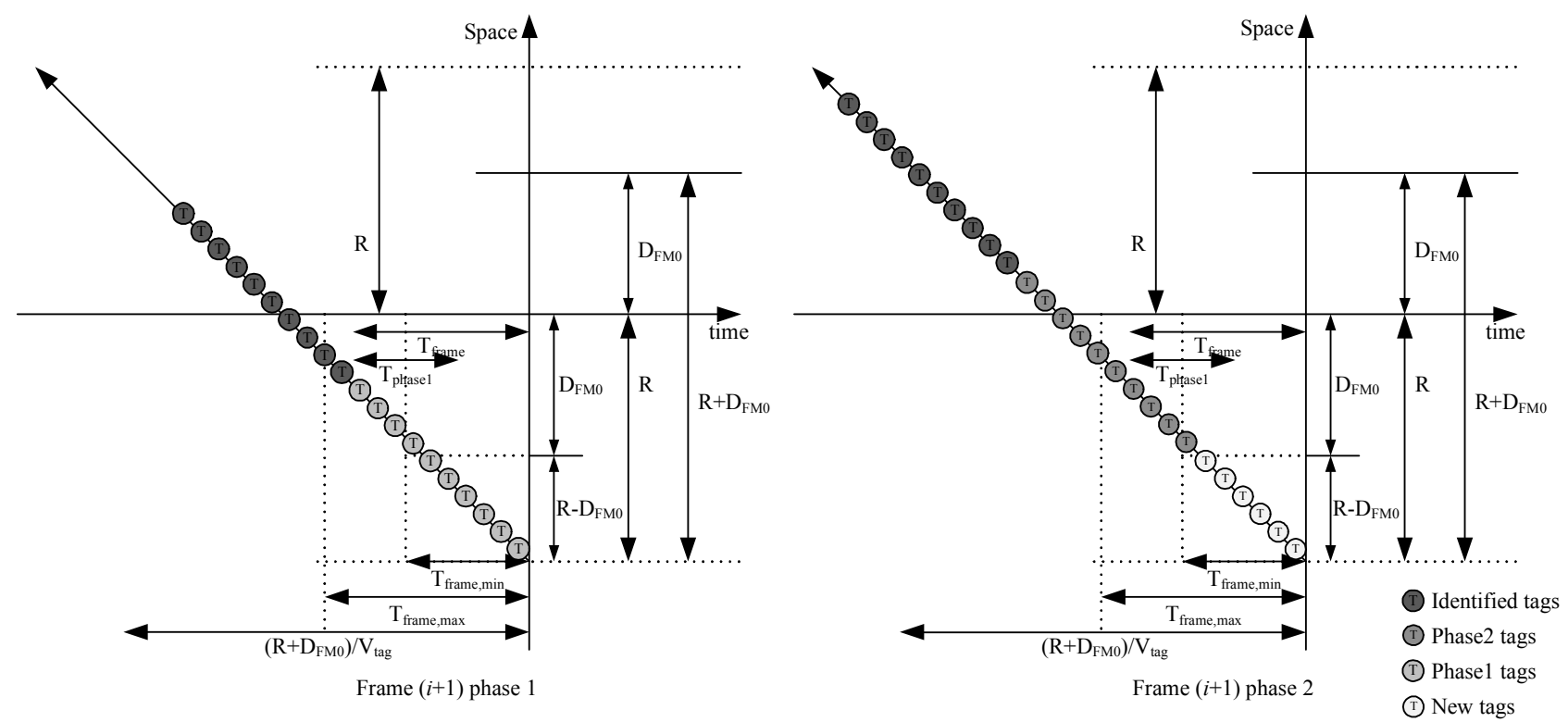

Fig. 5. An example of the proposed protocol at the $(i+1)$ th frame.

\section{Performance Analysis}

We analyze the identification rate of the proposed anti-collision protocol. In our proposed protocol, there is no collision at Phase 2. So we assume that there is no identification failure in Phase 2. Thus the probability of failure in Phase 1 is the overall probability of failure. In Phase 1 , sub-frame $j$ has $L_{j}$ slots, then the size of sub-frame $j$ is

$$
T_{\text {sub-frame }, j}=L_{j} \times T_{\text {slot-phase } 1}
$$

where $T_{\text {slot-phase } 1}$ is the size of a slot in Phase 1 . The number of slots in sub-frame $j$ is the same as the number of tags to be identified. The number of tags to be identified in sub-frame $j$ is the number of tags which collided $j$ times before sub-frame $j$. 


$$
L_{j}=\left\lceil\left(P_{\text {coll }}\right)^{j} \times\left(T_{\text {frame }} \times V_{\text {tag }} \times D_{\text {tag }}\right)\right\rceil
$$

where $P_{\text {coll }}$ is the probability that a collision occurs in a slot, and $\lceil x\rceil$ is the smallest integer greater than or equal to $x$. The maximum sub-frame index $j^{*}$ in Phase 1 is

$$
j^{*}=\arg \min _{j}\left\{\left(T_{\text {Phase } 1}-\sum_{l=1}^{j} T_{\text {sub-frame }, l}\right)>0\right\}
$$

Thus the probability $P_{\text {fail, }}$ prop that a tag fails in Phase 1 is

$$
P_{\text {fail,prop }}=\left(P_{\text {coll }}\right)^{j^{*}}
$$

We also analyze the identification rate of the conventional DFSA protocol. We assume an ideal DFSA protocol, i.e., the frame size is ideally decided as the number of tags. The frame size of the DFSA protocol $T_{\text {frame-DFSA }}$ is

$$
T_{\text {frame-DFSA }}=L_{D F S A} \times T_{\text {slot-DFSA }},
$$

where $L_{D F S A}$ is the number of slots in a frame and $T_{\text {slot-DFSA }}$ is the size of a slot in the DFSA protocol. $L_{D F S A}$ includes the number of new tags come into the identification range and the tags collided $k$ times. So $L_{D F S A}$ is

$$
L_{D F S A}=\sum_{k=0}^{C_{\max }-1} n(k)
$$

where $C_{\text {max }}$ is the number of collisions (frames) before the tags go out of the identification range of a reader,

$$
C_{\max }=\left\lceil\left(2 R / V_{\text {tag }}\right) / T_{\text {frame-DFSA }}\right\rceil \text {, }
$$

and $n(k)$ is the number of tags which collided or failed $k$ times due to channel error

$$
n(k)=\left(\prod_{i=0}^{k-1}\left(P_{\text {coll }}+P_{\text {succ }} \times P E R_{i}\right)\right) \times\left(T_{\text {frame-DFSA }} \times V_{\text {tag }} \times D_{\text {tag }}\right),
$$

where $P_{\text {succ }}$ is the probability that a slot is successful, and $P E R_{i}$ is the PER of the tags which fail $i$ times. So the distance between a reader and the tag which fail $i$ times is $\left|R-V_{\text {tag }} \cdot i \cdot T_{\text {frame-DFSA }}\right|$. Thus $P E R_{i}$ is

$$
P E R_{i}=P E R\left(\left|R-V_{\text {tag }} \times i \times T_{\text {frame }-D F S A}\right|\right) .
$$


Finally, the probability that a tag fails is

$$
P_{\text {fail }, D F S A}=\prod_{i=0}^{C_{\max }-1}\left(P_{\text {coll }}+P_{\text {succ }} \times P E R_{i}\right) .
$$

\section{Simulation Results}

We evaluate the performance of our proposed protocol. The parameters of simulations are presented in Table 1. Fig. 6 shows that the simulation results are closely matched with the analysis. The identification rate of our proposed anti-collision protocol $\left(P E R_{\text {req }}=10^{-4}\right)$ achieves $100 \%$ when $D_{\text {tag }}$ is less than 300 . The identification rate of our proposed anti-collision protocol with $P E R_{\text {req }}=10^{-3}$ is little lower than $100 \%$ but more reliable than the case of $P E R_{\text {req }}=10^{-4}$ for high density condition. When $P E R_{\text {req }}=10^{-5}$, the identification rate decreases more rapidly than the case of $P E R_{\text {req }}=10^{-4}$. The identification rate of DFSA does not achieve $100 \%$ even at low densities, since the tags in DFSA contend each other regardless of the orders of tag arrivals to the reader's identification range. DFSA uses a fixed line code (Miller $M=4$ ), so the identification speed is lower than that of our proposed anti-collision protocol.

Table 1. Simulation Parameters

\begin{tabular}{cc}
\hline \hline Parameters & Value \\
\hline Density of tags $\left(D_{\text {tag }}\right)$ & $50 \sim 500$ tags $/ \mathrm{m}$ \\
Speed of tags $\left(V_{\text {tag }}\right)$ & $1 \mathrm{~m} / \mathrm{s}$ \\
Required PER $\left(P E R_{\text {req }}\right)$ & $10^{-3}, 10^{-4}$, and $10^{-5}$ \\
Slot size of Phase $1\left(T_{\text {slot-phase } 1}\right)$ & $1 \mathrm{~ms}$ \\
Slot size of Phase $2\left(T_{\text {slot-phase } 2}\right)$ & $1 \mathrm{~ms}$ \\
Slot size of DFSA $\left(T_{\text {slot-DFSA }}\right)$ & $4 \mathrm{~ms}$ \\
Guard time $\left(T_{\text {guard }}\right)$ & $20 \mathrm{~ms}$ \\
Reader's reading range $(R)$ & $15 \mathrm{~m}$ \\
\hline \hline
\end{tabular}

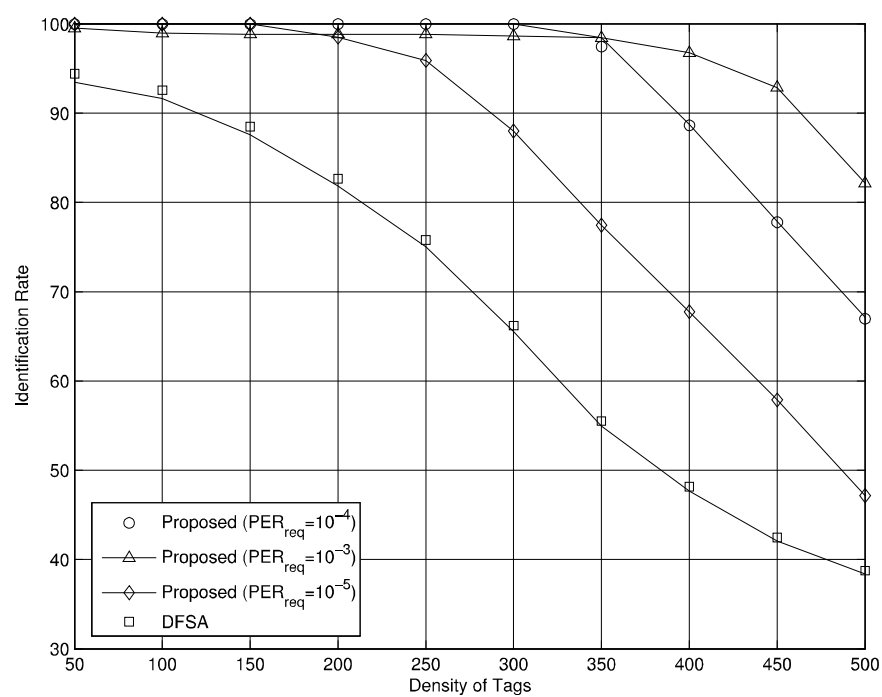

Fig. 6. The identification rate for varying density of tags (Analysis: Solid lines, simulations: Legends).

\section{Conclusion}

We have proposed a new RFID anti-collision protocol for moving tags under error-prone channel. The proposed anti-collision protocol can improve the identification rate of RFID tags by controlling the 
identification time depending on the varying channel condition due to the movement of tags. We have also proposed an appropriate frame size to maximize the identification rate. Performance analysis and simulation results indicate that the proposed anti-collision algorithm can achieve high identification rate compared to DFSA. Hence the reliability of an RFID monitoring system can be improved by applying our proposed anti-collision protocol.

\section{Acknowledgment}

This work was supported by the National Research Foundation of Korea (NRF) grant funded by the Korean government (MSIP) (2014R1A5A1011478).

\section{References}

[1] Simon, M., \& Divsalar, D. (2006). Some interesting observations for certain line codes with application to RFID. IEEE Transactions on Communications, 54(4), 583-586.

[2] Arnitz, D., Muehlmann, U., \& Witrisal, K. (2012). Characterization and modeling of UHF RFID channels for ranging and localization. IEEE Transactions on Antennas and Propagation, 60(5), 2491-2501.

[3] Nikitin, P. V., \& Rao, K. S. (2006). Theory and measurement of backscattering from RFID tags. IEEE Antennas and Propagation Magazine, 48(6), 212-218.

[4] Safarian, A., Shameli, A., Rofougaran, A., Rofougaran, M., \& Flaviis, F. de (2009). RF identification (RFID) reader front ends with active blocker rejection. IEEE Transactions on Microwave Theory and Techniques, 57(5), 1320-1329.

[5] Kim, D. Y., Jang, B. J., Yoon, H. G., Park, J. S., \& Yook, J. G. (2007). Effects of reader interference on the RFID interrogation range. Proceedings of European Conference on Wireless Technology (pp. 728-731). Munich, Germany.

[6] Krigslund, R., Popovski, P., Pedersen, G. F., \& Olesen, K. (2012). Interference helps to equalize the read range and reduce false positives of passive RFID tags. IEEE Transactions on Industrial Electronics, 59(12), 4821-4830.

[7] Yeo, S. M., Jeon, B. W., Bae, J. H., et al. (2008). A channel allocation scheme considering with collisions and interferences in practical UHF RFID applied communication fields. Proceedings of IEEE International Conference on RFID (pp. 258-268). Las Vegas, Nevada, USA.

[8] Eom, J. B., Yim, S. B., \& Lee, T. J. (2009). An efficient reader anticollision algorithm in dense RFID networks with mobile RFID readers. IEEE Transactions on Industrial Electronics, 56(7), 2326-2336.

[9] EPCglobal. (2005). EPCTM Radio-Frequency Identification Protocols Class-1 Generation-2 UHF RFID Protocol for Communications at 860MHz-960MHz Version 1.0.9.

[10] Fyhn, K., Jacobsen, R. M., Popovski, P., \& Larsen, T. (2012). Fast capture - Recapture approach for mitigating the problem of missing RFID tags. IEEE Transactions on Mobile Computing, 11(3), 518-528.

[11] Popovski, P., Fyhn, K., Jacobsen, R. M., \& Larsen, T. (2011). Robust statistical methods for detection of missing RFID tags. IEEE Wireless Communications, 18(4), 74-80.

[12] Luo, W., Chen, S., Li, T., \& Chen, S. (2011). Efficient missing tag detection in RFID systems. Proceedings of IEEE INFOCOM (pp. 356-360). Shanghai, China.

[13] Vogt, H. (2002). Efficient object identification with passive RFID tags. Pervasive Computing, 2414, 98-113.

[14] Lee, S. R., Joo, S. D., \& Lee, C. W. (2005). An enhanced dynamic framed slotted ALOHA algorithm for RFID tag identification. Proceedings of MobiQuitous (pp. 166-172). Boston, Massachusetts, USA.

[15] Prodanoff, Z. G. (2010). Optimal frame size analysis for framed slotted ALOHA based RFID networks. Computer Communications, 33(5), 648-653. 
[16] Xie, L., Sheng, B., Tan, C. C., Han, H., Li, Q., \& Chen, D. (2010). Efficient tag identification in mobile RFID systems. Proceedings of INFOCOM (pp. 1-9). San Diego, California, USA.

[17] Nakano, H., \& Sasabe, M. (2007). High-speed collective readout of large quantities of moving electronic tags using the response probability control method. IEEE Systems Journal, 1(2), 160-167.

[18] Park, J., \& Lee, T.-J. (2011). Channel-aware line code decision in RFID. IEEE Communications Letters, 15(12), 1402-1404.

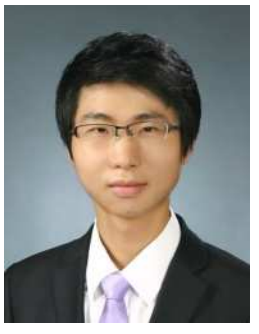

Ji Hyoung Ahn received the B.S. and M.S. degrees in electronic, electrical, and computer engineering from Sungkyunkwan University, Korea, in 2010 and 2012, respectively. He is currently working toward the Ph.D. degree in the College of Information and Communication Engineering at Sungkyunkwan University since March 2012. His research interests include medium access control (MAC) of RFID, wireless LAN, and wireless PAN.

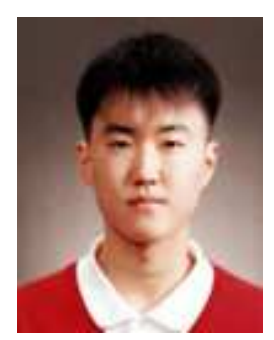

Jongho Park received the B.S., M.S. and Ph.D. degrees in electronic, electrical, and computer engineering from Sungkyunkwan University, Korea, in 2004 and 2006, 2012, respectively. He is now with the LG Electronics, Korea. His research interests include medium access control (MAC) of RFID, wireless communication networks, wireless LAN, wireless PAN, and Ad-Hoc networks.

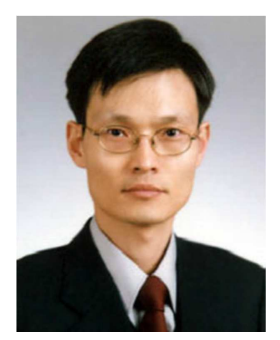

Tae-Jin Lee received his B.S. and M.S. degrees in electronics engineering from Yonsei University, Korea in 1989 and 1991, respectively, and the M.S.E. degree in electrical engineering and computer science from University of Michigan, Ann Arbor, in 1995. He received the Ph.D. degree in electrical and computer engineering from the University of Texas, Austin, in May 1999. In 1999, he joined the Corporate R \& D Center, Samsung Electronics where he was a senior engineer. Since 2001, he has been a professor in the College of Information and Communication Engineering at Sungkyunkwan University, Korea. He was a visiting professor in Pennsylvania State University from 2007 to 2008. His research interests include performance evaluation, resource allocation, medium access control (MAC), and design of communication networks and systems, wireless LAN/PAN/MAN, ad-hoc/sensor/RFID networks, next generation wireless communication systems. He has been a voting member of IEEE 802.11 WLAN Working Group, and is a member of IEEE and IEICE. 